\title{
FREQUÊNCIA DE OCORRÊNCIA DE Giardia sp. E DE Cryptosporidium spp. EM EQUÍDEOS NATURALMENTE INFECTADOS E ABATIDOS EM UM FRIGORÍFICO
}

\author{
NAYARA RESENDE NASCIUTTI ${ }^{1}$, DAGMAR DINIZ CABRAL $^{2}$, ALINE DINIZ CABRAL ${ }^{3}$, RAFAEl ROCHA DE \\ SOUZA $^{4}$, PATRICIA MAGALHÃES DE OLIVEIRA ${ }^{4}$
${ }^{1}$ Mestre em Ciências Veterinárias pela Universidade Federal de Uberlândia, Uberlândia, MG, Brasil. nayaranasciutti@yahoo.com.br
${ }^{2}$ Professora Mestre da Universidade Federal de Uberlândia, Uberlândia, MG, Brasil. ${ }^{3}$ Doutoranda da Universidade de São Paulo, São Paulo, SP. Brasil.
${ }^{4}$ Residentes Médicos Veterinários da Universidade Federal de Uberlândia, Uberlândia, MG, Brasil.

\begin{abstract}
Dentre os protozoários de maior importância em saúde pública estão Cryptosporidium spp. e Giardia sp. pelo potencial zoonótico. Pesquisou-se a frequência de ocorrência desses parasitas em equídeos abatidos em um frigorífico no município de Araguari, Minas Gerais, durante o período de fevereiro a março de 2008, correlacionando com o sexo, espécie e procedência dos animais. Foram coletadas 150 amostras de fezes, utilizando-se técnicas coproparasitológicas específicas para a detecção de Cryptosporidium spp. e Giardia sp. Os resultados revelaram a presença de cistos de Giardia sp. em $4 \%(6 / 150)$ das amostras. A porcentagem de fêmeas positivas foi $4,23 \%$ (3/71) e machos 3,80\% (3/79). Entre

os equídeos a porcentagem de positivos foi 10,53\% (2/19) em muares e 3,05\% (4/131) em equinos. Quanto à procedência dos animais positivos por estados brasileiros, a frequência foi $16,67 \%$ (1/6) na Bahia; 7,69\% (1/13) em Tocantins; 3,61\% (3/83) em Minas Gerais e 2,08\% (1/48) em Goiás. Oocistos de Cryptosporidium spp. não foram encontrados em nenhuma das 150 amostras de fezes analisadas. Conclui-se que é importante estudar a frequência desses protozoários em equídeos, acrescentando dados à literatura, bem como sugerir estudos moleculares para pesquisar o genótipo circulante e, desse modo, associar com a epidemiologia desses protozoários e a saúde pública.
\end{abstract}

PALAVRAS-CHAVE: cryptosporidiose; equídeos; giardíase; protozoonoses.

FREQUENCY OF OCCURRENCE OF Giardia sp. AND Cryptosporidium spp. IN
EQUIDAE NATURALLY INFECTED AND SLAUGHTERED IN A SLAUGHTER
HOUSE

\section{ABSTRACT}

Cryptosporidium spp. and Giardia sp are protozoan of larger importance by their zoonotic potential. We studied the frequency of occurrence of these parasites in equidae from Araguari - Minas Gerais, slaughtered during February to March of 2008 and correlated with sex, species and origin. A total of 150 fecal samples were collected and specific techniques were used. The results showed 4\% (6/150) of Giardia sp. in all the samples. The positivity was $4.23 \%(3 / 71)$ in females and $3.80 \%(3 / 79)$ in males. The positivity was $10.53 \%(2 / 19)$ in mules and $3.05 \%$ (4/131) in horses. The positive samples from Brazilian states was $16.67 \%(1 / 6)$ in Bahia, $7.69 \%(1 / 13)$ in Tocantins, 3.61\% (3/83) in Minas Gerais and 2.08\% (1/48) in Goiás. Cryptosporidium spp. were not found in any samples analyzed $(n=150)$. We concluded that it is very important to study the frequency of these protozoans in horses, adding data to the; we also suggest molecular studies to associate with epidemiology and public health.

KEYWORDS: cryptosporidiosis; giardiasis; horses; protozoal. 


\section{INTRODUÇÃO}

Cryptosporidium spp. e Giardia sp. são atualmente reconhecidos como os principais patógenos entéricos com potencial zoonótico (GOMES et al., 2008), podendo causar diarreia, dor abdominal, náusea e vômito (GEURDEN et al., 2010, ANKARKLEV et al., 2010). ETTHAD et al. (2010) relataram que existe relação entre o crescimento retardado em crianças e diarreia persistente com infecções de Cryptosporidium spp. e Giardia sp, entre outros patógenos. A transmissão desses protozoários pode ocorrer pela via fecal-oral ou pela ingestão de água e alimentos contaminados (JOHNSON et al., 1997).

A Giardia sp. possui dois estágios, trofozoíto (forma vegetativa) e cisto (forma infectante) (LYONS et al., 1991), e pode causar má absorção, hipersecreção aniônica e aumento do transito intestinal, resultando em diarreia (COTTON et al., 2011). Os equinos também podem apresentar esses sinais, porém a presença do cisto nas fezes não indica que os animais estejam doentes (LYONS et al., 1991). Outras espécies também são susceptíveis como os animais de estimação, ruminantes e animais selvagens. Provavelmente a maioria das infecções são assintomáticas (BURET, 2007).

No Brasil, há relato de eliminação de cistos de Giardia sp. por equinos do noroeste e do norte do estado do Paraná, sendo a ocorrência estimada em 21,1\% (26/123) (VARGAS \& RIGOLON, 1998). No estado do Rio de Janeiro, SOUZA et al. (2009) detectaram cistos de Giardia apenas em um grupo, em que dois dos 30 equinos estavam parasitados (6,67\%). do total de 396 amostras individuais, apenas $0,5 \%$ dos equinos estudados estavam infectados.

Oocistos de Cryptosporidium spp. são eliminados nas fezes e quando ingeridos, eliminam os esporozoítos que invadem as microvilosidades intestinais (LYONS et al., 1991). Esse parasito é reconhecido como um microorganismo patogênico que causa diarreia aquosa e perda de peso (JOHNSON et al., 1997).

O contato direto durante o trabalho ou a recreação com equídeos infectados por Giardia sp. e Cryptosporidium spp. podem constituir uma fonte importante de infecção para seres humanos (TRAUB et al., 2005). GOMES et al. (2008) afirmaram que, como os dados ainda são escassos, é preciso buscar mais informações a respeito prevalência desses protozoários na espécie equina. Neste contexto, o objetivo do trabalho foi verificar a frequência de ocorrência de Giardia sp. e
Cryptosporidium spp. em equídeos abatidos em um frigorífico em Araguari-MG, correlacionando com o sexo, espécie e procedência dos animais.

\section{MATERIAL E MÉTODOS}

O trabalho foi realizado no Frigorífico Pomar de Araguari-MG, especializado no abate de equídeos provenientes de diferentes estados, durante o período de fevereiro a março de 2008. Coletaram-se aleatoriamente, no setor de triparia, 150 amostras de fezes diretamente do cólon intestinal dos equídeos com o auxílio de sacos plásticos descartáveis, que eram devidamente identificados com o mesmo número marcado no intestino grosso, possibilitando o rastreamento dos dados dos animais.

As fezes colhidas foram transportadas em caixas de isopor e levadas sob refrigeração para o laboratório de protozoologia da Universidade Federal de Uberlândia (UFU) onde foram processadas.

A técnica de FAUST et al. (1938) foi utilizada para identificação dos cistos de Giardia sp. sob microscopia ótica (400X), sendo realizada no máximo em 24 horas após a colheita. O restante das amostras foi armazenado em formalina $10 \%$ para posterior realização de esfregaços de fezes, fixados com metanol, corados pela técnica de Ziehl-Nielsen modificada (HENRIKSEN \& POHLENS, 1981), para visualização dos oocistos de Cryptosporidium spp.

Para análise estatística dos resultados, utilizou-se o teste binomial para duas proporções (AYRES et al., 2005).

\section{RESULTADOS E DISCUSSÃO}

Dos 150 animais analisados para a presença de Giardia sp., seis apresentaram cistos nas fezes, representando 4,0 \%. Esse resultado foi superior aos encontrados por SOUZA et al. (2009), que observaram uma positividade de $0,5 \%(2 / 396)$ em equinos naturalmente infectados no estado do Rio de Janeiro, e por GOMES et al. (2008), que não observaram cisto de Giardia sp. nas amostras de fezes de 66 equinos com idade entre dois e 12 anos, estabulados no Jockey Club de Santa Maria - RS. HEIN et al. (2012) verificaram a ocorrência de $3,33 \%$ (1/30) de Giardia sp. em amostras de 30 equinos provenientes da Vila Osternack em Curitiba-PR.

Embora a infecção por Giardia sp. em equinos seja considerada rara (XIAO \& HERD, 1994), a frequência de ocorrência da giardíase 
estimada no trabalho de VARGAS \& RIGOLON (1998) foi de $21,1 \%$ (26/123), mostrando que essa infecção é relativamente comum em equinos da região do noroeste e norte do Paraná.

Outros estudos no exterior têm revelado a prevalência de Giardia sp. em 3,2\% dos cavalos utilizados para recreação na Califórnia (JOHNSON et al., 1997), 4,6\% em cavalos e mulas usados como transporte em Nevada, Estados Unidos (ATWILL et al., 2000) e 20\% em fazendas no Canadá (OLSON et al., 1997).

No presente estudo, a frequência da infecção por Giardia sp. foi semelhante em fêmeas 4,23\% (3/71) e machos 3,80\% (3/79), não havendo diferença estatística quanto ao sexo dos animais, corroborando o trabalho de VARGAS \& RIGOLON (1998), que encontraram positividade em $20,5 \%$ das fêmeas e $22,0 \%$ dos machos (Tabela $1)$.

Tabela 1- Frequência de ocorrência de cistos de Giardia sp. em relação ao sexo dos equídeos naturalmente infectados, abatidos no Frigorífico de Araguari-MG, 2008

\begin{tabular}{lccc}
\hline Sexo & Examinados & Positivos & $\%$ \\
\hline Macho & 79 & 3 & $3,80^{\mathrm{a}}$ \\
Fêmea & 71 & 3 & $4,23^{\mathrm{a}}$ \\
\hline Total & 150 & 6 & 4,00 \\
\hline
\end{tabular}

Letras minúsculas diferentes diferem estatisticamente $(\mathrm{P}<0,05)$

No presente trabalho, a positividade em muares, $10,53 \%$ (2/19), foi maior que nos equinos, $3,05 \%$ (4/131), mas não houve diferença estatística (Tabela 2). Porém, é um dado importante, pois não foi encontrada na literatura brasileira a descrição de cistos de Giardia sp. em muares.

A Tabela 3 mostra que a maior frequência de cisto de Giardia sp. foi em animais oriundos do estado da Bahia, apesar de não haver diferenças estatísticas entre os demais estados. Dos animais positivos, a frequência foi de 2,08\% (1/48) em Goiás, 3,61\% (3/83) em Minas Gerais, 7,69\% (1/13) em Tocantins e 16,67\% (1/6) na Bahia.

Tabela 2- Frequência de ocorrência de cistos de Giardia sp. em relação à espécie dos equídeos naturalmente infectados, abatidos no Frigorífico de Araguari-MG, 2008

\begin{tabular}{llll}
\hline Espécie & Examinados & Positivos & $\%$ \\
\hline Equina & 131 & 4 & $3,05^{\mathrm{b}}$ \\
Muar & 19 & 2 & $10,53^{\mathrm{b}}$ \\
\hline Total & 150 & 6 & 4,00 \\
\hline
\end{tabular}

Letras minúsculas diferentes diferem estatisticamente $(\mathrm{p}<0,05)$

Tabela 3- Frequência de cistos de Giardia sp. por estados brasileiros dos equídeos naturalmente infectados, abatidos em um Frigorífico de Araguari-MG, 2008

\begin{tabular}{llll}
\hline Estados & Examinados & Positivos & $\%$ \\
\hline Goiás & 48 & 1 & $2,08 \mathrm{c}$ \\
Minas Gerais & 83 & 3 & $3,61 \mathrm{c}$ \\
Tocantins & 13 & 1 & $7,69 \mathrm{c}$ \\
Bahia & 6 & 1 & $16,67 \mathrm{c}$ \\
\hline Total & 150 & 6 & 4,00 \\
\hline
\end{tabular}

Letras minúsculas diferentes diferem estatisticamente $(\mathrm{p}<0,05)$ 
Os resultados no presente estudos devem ser considerados como uma subestimativa, em virtude dos equinos eliminarem cistos de Giardia sp. de forma intermitente (XIAO \& HERD, 1994) e da possibilidade de alguns animais se encontrarem no período prepatente da infecção (VARGAS \& RIGOLON, 1998).

Cerca de $7 \%$ da população humana mundial abriga Giardia sp. no intestino delgado, mas pouco se conhece sobre a epidemiologia desse microorganismo, sobretudo com referência ao possível papel de outros mamíferos servirem como fonte de infecção humana. Os cistos de Giardia sp. são comumente encontrados em fezes de cães, gatos, bovinos, ovinos, caprinos, lhamas e de outros mamíferos domésticos e selvagens, embora a transmissão cruzada entre os hospedeiros possa ocorrer, pouco se sabe sobre essa frequência de ocorrência (PALMER et al., 2008).

De acordo com THOMPSON (2004), mais de 50 espécies de Giardia já foram descritas, mas atualmente são reconhecidas apenas cinco espécies: G. duodenalis, G. agilis, G. muris, G. ardeae e G. psittaci. A espécie G. agilis parasita anfíbios; $G$. muris parasita roedores; G. ardeae parasita aves; $G$. psittaci parasita aves e a espécie $G$. duodenalis parasita vários mamíferos domésticos e selvagens, incluindo humanos.

Novos grupos chamados assemblages foram determinados, sendo sete para G. duodenalis (A, B, C, D, E, F e G), cuja taxonomia ainda está em discussão e os cistos são indistinguíveis morfologicamente, mas podem ser diferenciados molecularmente (THOMPSON et al., 2000; CACCIO \& RYAN, 2008). Apenas os assemblages A e B têm sido detectados em humanos e em outros mamíferos, sendo considerados potencialmente zoonóticos, havendo necessidade de mais estudos (SPRONG et al., 2009).

No presente estudo, não foram encontrados oocistos de Cryptosporidium spp. nas 150 amostras de fezes analisadas. Esse resultado corrobora o trabalho de JOHNSON et al. (1997), que analisaram 91 amostras fecais e não encontraram cistos de Giardia sp. e nem oocistos de Cryptosporidium spp. em equinos de recreação na Califórnia.

SOUZA et al. (2009) pesquisaram 396 amostras fecais de equinos, encontrando oocistos de Cryptosporidium spp. em três amostras, representando 0,75\%, no Rio de Janeiro. Em um estudo realizado no Rio Grande do Sul, SILVA et al. (1996) relataram a ocorrência de oocistos de Cryptosporidium spp. em $40 \%$ das amostras analisadas $(\mathrm{n}=10)$. Em outro estudo, GOMES et al. (2008) encontraram o parasito em $75 \%$ das amostras, sendo este alto índice justificado por uma possível contaminação hídrica no Jockey Club em Santa Maria- RS.

TOSCAN et al. (2010) compararam a prevalência de Cryptosporidium spp. em equinos de tração e em atletas do Jockey Club de Santa Maria (RS) e encontraram nos 104 animais, 38,5\% (20/52) e $80,8 \%$ (42/52), respectivamente.

De acordo com OLSON et al. (1997), a frequência de infecções de Cryptosporidium spp. em equinos é pouco conhecida e acredita-se que a maioria dos infectados sejam portadores assintomáticos. Para se iniciar estudos epidemiológicos de infecção cruzada, seria necessário proceder com a caracterização genotípica de Cryptosporidium spp. e Giardia spp. em amostras de campo de animais naturalmente infectados e, assim, sugerir hipóteses para determinar a origem dos parasitas, os hospedeiros com importância zoonótica e as principais fontes de infecção, tanto para animais quanto para o ser humano, estabelecendo medidas preventivas.

\section{AGRADECIMENTOS}

À Professora Dagmar Diniz Cabral pelo incentivo e dedicação, à Fundação de Amparo à Pesquisa de Minas Gerais (FAPEMIG) pelo auxílio ao projeto, ao Médico Veterinário Valmir Tunala e equipe do Frigorífico Pomar de Araguari-MG pelo apoio, à técnica do laboratório de parasitologia da Universidade Federal de Uberlândia, Elaine Silva Marques Faria. Às biólogas Rúbia Mara Rodrigues Amorim e Maria Júlia Rodrigues Cunha. E aos amigos acadêmicos do curso de Medicina Veterinária da Universidade Federal de Uberlândia (UFU), Rafael Rocha de Souza e Gabriel Freitas Martins.

\section{REFERÊNCIAS}

ANKARKLEV, J.; JERLSTROM-HULTQVIST, J.; RINGQVIST, E.; TROELL K.; SVARD S.G. Behind the smile: cell biology and disease mechanisms of giardia species. Nature Reviews Microbiology. v.8, p.413-22, 2010.

ATWILL, E.R.; MECDOUGALD, N.K.; PEREA, L. Cross-sectional study of faecal shedding of Giardia duodenalis and Cryptosporidium parvum among packstock in the Sierra Nevada Range. Equine Veterinary Journal, v.32, n. 3, p.247-252, 2000.

AYRES, M.; AYRES Jr, M.; AYERS, D.L.; SANTOS, A. S. S. BioEstat 5.0: Aplicações estatísticas nas áreas das ciências biológicas e médicas. Belém: Sociedade Civil Mamirauá; Brasília; DF; Conselho Nacional de Desenvolvimento Científico e Tecnológico (CNPq), 2005. $324 \mathrm{p}$. 
BURET, A.G., Mechanisms of epithelial dysfunction in giardiasis. Gut, v. 56, p. 316-317, 2007.

CACCIO, S. M.; RYAN, U. Molecular epidemiology of giardiasis. Molecular and Biochemical Parasitology, v. 160, n. 2, p. 75-80, 2008.

COTTON, J. A.; BEATTY, J. K.; BURET, A.G. Host parasite interactions and pathophysiology in Giardia infections. Internation Journal Parasitology, v. 41, p. 929-933, 2011.

ETTEHAD, G.H.; DARYANI, A.; NEMATI, A. Effect of Giardia infection on nutritional status in primary schoolchildren, in Northwest Iran. Pakistani Journal Biological Sciences. v. 13, n.5, p 229-234, 2010.

FAUST, E.C.; D`ANTONI, I.C.; ODON, V.; MILLER, M. J.; PERES, C.; SAWITZ, W.; THOMEN, L. F.; TOBIE, J.; WALKER, J. H. A critical study of clinical laboratory techniques for the diagnosis of protozoan cysts and helminth eggs in feces. I. Preliminary communication. American Journal of Tropical Medicine and Hygiene, v. 18, p. $169-183,1938$.

GOMES, A.D.; BARRETA, C.; ZIEGLER, D.P.; SAUSEN, L.; STOEVER, N.; SANGIONI, L.A.; VOGEL, F.F.; MONTEIRO, S.G.; ZANELLA, A. Prevalência de Cryptosporidium and Giardia sp em eqüinos estabulados no Jockey Club de Santa Maria- RS, Brasil. Ciência Rural, v.38, n.9, p.2662-2665, 2008.

GEURDEN, T.; VERCRUYSSE, J.; CLAEREBOUT, E. Is Giardia a significant pathogen in production animals? Experimental Parasitology. v. 124, p.98-106, 2010.

HEIN, K.K.; YOKOYOMA, M.R.; SCHAULE, M.T.; BUDEL, G.D.; NOGARI, F.; GONÇALVES, D.; FUNCHAL, E. Verificação da ocorrência parasitológica com potencial zoonótico em fezes de equinos na Vila Osternack- Curitiba-PR. Revista Eletrônica da Faculdade Evangélica do Paraná. v. 2, n.2, p.71-79, 2012.

HENRIKSEN, S.A.; POHLENS, J. Staining of Cryptosporidia by modified Ziehl-Neelsen technique. Acta Veterinaria Scandinavic. v.22, p.594-596, 1981.

JOHNSON, E.; ATWILL, E.R.; FILKINS, M.E.; KALUSH, J. The prevalence of shedding of Cryptosporidium and Giardia spp.based on a single fecal sample collection from each of 91 horses used for backcountry recreation. Journal of Veterinary Diagnostic Investigation, v.9, p.56-60, 1997.

LYONS, E.T.; GRANSTROM, D.E.; DRUDGE, J.H.;
TOLLIVER, S.C. The role of intestinal protozoa in foal diarrhea. Veterinary Medicine, v. 86, n.2, p. 193-197, 1991.

OLSON, B.E.; THORLAKSON, C.L.; DESELLIERS, L.; MORCK, D.W.; McALLISTER, T.A. Giardia and Cryptosporidium in Canadian farm animals. Veterinary Parasitology, v.68, n.4, p.375-381, 1997.

PALMER, C. S.; TRAUB, R. J.; ROBERTSON, I. D.; DEVLIN, G.; REES, R.; THOMPSON, R. C. Determining the zoonotic significance of Giardia and Cryptospordium in Australian dogs and cats. Veterinary Parasitology, v. 154, n. 1-2, p. 142-147, 2008.

SILVA，N.R.S.; BRACCINI，G.L.; CHAPLIN， E.L.; ARAÚJO, F.A.P. Infecção mista por Cryptosporidium parvum e C. muris em eqüinos de Porto Alegre, RS, Brasil. Arquivos da Faculdade de Veterinária UFRGS, v.24, n.1 p. 81-84, 1996.

SOUZA, P.N.B de.; BOMFIM, T.C.B. ; HUBER, F.; ABBOUD, L.C.S.; GOMES, R.S. Natural infection by Cryptosporidium sp., Giardia sp. and Eimeria leuckarti in three groups of equines with different handlings in Rio de Janeiro, Brazil. Veterinary Parasitology, v.160, p.327333, 2009.

SPRONG, H.; CACCIO, S. M.; VAN DER GIENSSEN, J. W. Identification of zoonotic genotypes of Giardia duodenalis. PLOS Neglected Tropical Diseases, v. 3, n. 12, p. e558, 2009.

TOSCAN, G.; PEREIRA,R.C.F; ARAUJO,L.; SANGIONI, L.A.; VOGEL, F.S.F. Comparação da prevalência de Cryptosporidium spp. em equinos de tração e em atletas do Jockey Club de Santa Maria, RS, Brasil. Ciência Animal Brasileira, v.11, n.2, p.436-440, 2010.

THOMPSON, R.C.A.; HOPKINS, R.M.; HOMAN, W.I. Nomenclature and genetics groupings of Giardia infecting mammals. Parasitology Today, v.16, p.210-213, 2000.

TRAUB, R.J. ; WADE, S. ; READ, C.; THOMPSON, A.; MOHAMMED, H. Molecular characterization of potentially zoonotic isolates of Giardia duodenalis in horses. Veterinary Parasitology, v.130, p.317-321, 2005.

VARGAS, L; RIGOLON, L. P. Ocorrência de Giardia duodenalis em eqüinos do noroeste e do norte do Paraná. Semina Ciências Agrárias, v.19, n.1, p.73-75, 1998.

XIAO, L.; HERD, R.P. Epidemiology of equine Cryptosporidium and Giardia infections. Equine Veterinary Journal, v.26, p.14-17, 1994. 